\title{
RETRACTED ARTICLE: Experimental and Theoretical Studies of Ferroelectric, Magnetic, Structural and Electronic Properties of $\mathrm{Sr}_{2} \mathrm{TiMnO}_{6}$ Material
}

\author{
J. Arbey Rodríguez • Fabio Fajardo • \\ David A. Landínez Téllez • Jairo Roa-Rojas · \\ Paulo Pureur
}

Received: 21 November 2011 / Accepted: 11 January 2012 / Published online: 5 February 2012

(C) Springer Science+Business Media, LLC 2012

Withdrawn due to the previous publication by the authors of

a similar paper.

J. Arbey Rodríguez (凶) · F. Fajardo

GEMA-Grupo de Estudio de Materiales, Departamento de Física,

Universidad Nacional de Colombia, AA 5997 Bogotá DC,

Colombia

e-mail: jroar@unal.edu.co

D.A. Landínez Téllez · J. Roa-Rojas

Grupo de Física de Nuevos Materiales, Departamento de Física,

Universidad Nacional de Colombia, AA 5997 Bogotá DC,

Colombia

P. Pureur

Instituto de Física, Universidade Federal do Rio Grande do Sul,

P.O. Box 15051, 91501-970, Porto Alegre, RS, Brazil 